\title{
Krebs, Heinz
}

\section{Methodische Aspekte professionellen Handelns in Pädagogik und Sozialer}

\section{Arbeit}

Haubl, Rolf [Hrsg.]; Dammasch, Frank [Hrsg.]; Krebs, Heinz [Hrsg.]: Riskante Kindheit. Psychoanalyse und Bildungsprozesse. Göttingen : Vandenhoeck \& Ruprecht 2009, S. 204-220. - (Schriften des Sigmund-Freud-Instituts. Reihe 3: Psychoanalytische Sozialpsychologie; 004)

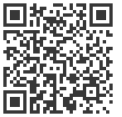

\section{Quellenangabe/ Reference:}

Krebs, Heinz: Methodische Aspekte professionellen Handelns in Pädagogik und Sozialer Arbeit - In: Haubl, Rolf [Hrsg.]; Dammasch, Frank [Hrsg.]; Krebs, Heinz [Hrsg.]: Riskante Kindheit. Psychoanalyse und Bildungsprozesse. Göttingen : Vandenhoeck \& Ruprecht 2009, S. 204-220 - URN:

urn:nbn:de:0111-pedocs-111010 - DOI: 10.25656/01:11101

\section{Vandenhoeck \& Ruprecht v\&R}

http://www.v-r.de

\section{Nutzungsbedingungen}

Gewährt wird ein nicht exklusives, nicht übertragbares, persōnliches und beschränktes Recht auf Nutzung dieses Dokuments. Dieses Dokument ist ausschließlich für den persönlichen, nicht-kommerziellen Gebrauch bestimmt. Die Nutzung stellt keine Übertragung des Eigentumsrechts an diesem Dokument dar und gilt vorbehaltlich der folgenden Einschränkungen: Auf sämtlichen Kopien dieses Dokuments müssen alle Urheberrechtshinweise und sonstigen Hinweise auf gesetzlichen Schutz beibehalten werden. Sie dürfen dieses Dokument nicht in irgendeiner Weise abändern, noch dürfen Sie dieses Dokument für öffentliche oder kommerzielle Zwecke vervielfältigen, öffentlich ausstellen, aufführen, vertreiben oder anderweitig nutzen.

Mit der Verwendung dieses Dokuments erkennen Sie die Nutzungsbedingungen an.

\section{Terms of use}

We grant a non-exclusive, non-transferable, individual and limited right to using this document.

This document is solely intended for your personal, non-commercial use. Use of this document does not include any transfer of property rights and it is conditional to the following limitations: All of the copies of this documents must retain all copyright information and other information regarding legal protection. You are not allowed to alter this document in any way, to copy it for public or commercial purposes, to exhibit the document in public, to perform, distribute or otherwise use the document in public.

By using this particular document, you accept the above-stated conditions of use.

\section{Kontakt / Contact:}

\section{peDOCS}

DIPF | Leibniz-Institut für Bildungsforschung und Bildungsinformation

Informationszentrum (IZ) Bildung

E-Mail: pedocs@dipf.de

Internet: www.pedocs.de

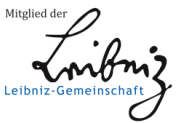




\section{Methodische Aspekte professionellen Handelns in Pädagogik und Sozialer Arbeit}

Das Verhältnis von Subjekt und Ort. Ungewissheit als methodisches Problem professionellen Handelns

Pädagogik und Soziale Arbeit sind gerade in den letzten Jahren ins Zentrum öffentlicher Diskussionen gerückt. "Bildung, Erziehung und Betreuung" (BMFSJ, 2005) sind zu einem voraussetzungsvollen Geschäft geworden. Eltern fühlen sich in der Erziehung verunsichert, weil sie nicht mehr auf ein solides Fundament verbindlicher Normen und Werte zurückgreifen können (Ahrbeck, 2004). Das Bildungssystem ist vom PISA-Schock erfasst und sieht sich dem Verdikt ausgesetzt, soziale Ungleichheit und Selektion herzustellen, anstatt Chancengleichheit und eine gute Bildung für alle Heranwachsenden zu gewährleisten (Deutsches PISA-Konsortium, 2001; Kerstan, 2004). Die Fachleute stehen oft ratlos vor diesen Krisenerscheinungen, die sich - um noch zwei weitere Beispiele zu nennen - im Niedergang der Hauptschule (Klemm, 2004) oder in den spektakulären Fällen dissozialer Entwicklungen von Heranwachsenden oder der Misshandlung von Kindern niederschlagen.

Vor diesem Hintergrund zeigt sich, dass professionelles Handeln in den Feldern der Sozialen Arbeit und Pädagogik komplexer und in seinen Wirkungen unkalkulierbarer geworden ist. Fachkräfte benötigen methodische Instrumentarien und Zugänge, um diese Ungewissheiten bewältigen zu können. Bei diesen Zugängen handelt sich um Formen, Stile und Routinen des Umgangs mit sozialen und psychischen Problemen. In einigen Fällen handelt es sich dabei auch um Eingriffe in das Leben Anderer, wie z. B. bei In-Obhutnahmen von Kindern. Allgemein gesehen ist es jedoch für diese Problembe- 
reiche charakteristisch, dass zwischen den methodischen Zugängen einerseits und dem tatsächlichen pädagogisch-professionellen Handeln andererseits kein eindeutiges Mittel-Zweck-Verhältnis besteht. Fachkräfte können ihre Handlungen in der Regel nicht in einer vollkommen kontrollierbaren Weise umsetzen. Methodische $\mathrm{Zu}$ gänge können insofern nur kritische Korrektive einer nur begrenzt methodisierbaren Praxis sein. Diese kann nicht aus Methoden und Theorien abgeleitet werden, sondern folgt einer Eigenlogik, die sich aus alltäglichen Lebenszusammenhängen, persönlichen Motiven und sozialen sowie institutionellen Zwängen speist. Diese Sicht auf pädagogisches Handeln bricht mit der Vorstellung der Möglichkeit einer direkten Anwendung von Wissensbeständen und Methoden auf die Praxis und unterstellt infolgedessen, dass die eigentlich professionelle Leistung in der reflexiven Verständigung über die individuellen Sinnzusammenhänge und Bedeutungsmuster in Kontexten von Bildung, Erziehung und Betreuung besteht (Müller, 2001; Hörster, 2002a).

Professionelles Handeln kann in der Moderne nicht mehr von seiner Ungewissheit befreit werden (Helsper, Hörster u. Kade, 2003). Es bedarf methodischer Zugänge und Reflexionshilfen, weil es folgende soziale, institutionelle und subjektbezogene Bedingungen bewältigen muss:

- Professionelles Handeln ist immer den existentiellen Bedingungen und dem konkreten Leben seiner Adressaten ausgesetzt, unabhängig davon, ob es in der Schule, in der Erziehungsberatung oder in der Jugendarbeit stattfindet. Methoden haben es in diesem Problemkreis mit der Aufgabe zu tun, Dialogbereitschaft herzustellen, soziale Nähe zu den Adressaten zu ermöglichen, d.h. Zugangsprobleme zu bewältigen, aber auch reflexive Distanz herzustellen (Müller, 1994, S. 11 ff.).

- Professionelle sind unvermeidlich mit der Gebrochenheit der eigenen Intentionen konfrontiert, da professionelles Handeln auf die selbsttätige Ausgestaltung von Zukunft und die Standortvergewisserung auf der Seite der Adressaten ausgerichtet ist. Besonders deutlich wird dies bei Heranwachsenden und damit beim Umgang mit dem Generationenverhältnis. Das heißt, die ältere Generation muss die Jüngere in das gegenwärtige Leben einführen, ohne $\mathrm{Zu}$ kunft zu verschließen. Pädagogik und soziale Arbeit sind insofern 
mit der Entwicklungstatsache konfrontiert. Im Rahmen des Generationenverhältnisses stellt sich dieser Umstand radikaler als beim Umgang mit erwachsenen Adressaten, dennoch kann zusammenfassend festgehalten werden, dass professionelles Handeln immer intersubjektives Handeln ist, das heißt abhängig vom Handeln einer oder mehrerer Personen. Methoden haben es insofern in diesem Problemkreis mit dem Umgang mit Ohnmacht, aber auch mit Macht bzw. bewusstem Machtverzicht zu tun (Müller, 2001).

- Aus dem Umstand der prinzipiellen Intersubjektivität erwächst die Forderung, dass Fachkräfte und Adressaten zusammenwirken müssen, ansonsten geschieht in Bildung, Erziehung und Betreuung gar nichts. Dabei muss aber bedacht werden, dass die Beteiligten nicht direkt zusammenwirken, sondern sich auf einen "dritten Faktor" (Winkler, 2002) beziehen müssen, in dem sich die soziokulturellen Bedingungen des gegenwärtigen Lebens niederschlagen, der aber auch die rechtlichen und institutionellen Regelungen einschließt, unter denen beispielsweise die Schule mit ihren Lehrplänen oder die Kinder- und Jugendhilfe mit dem Kinder- und Jugendhilfegesetz (SGB VIII, 2005) arbeiten. Müller (2001) spricht an diesem Punkt von den "auferlegten Relevanzen", die unabhängig von den beteiligten Personen gelten und denen sich diese - zumindest teilweise - anpassen müssen. Hier kommen methodische Ansätze zum Tragen, die es erlauben, sich in den sozialen, rechtlichen und institutionellen Abhängigkeiten zurechtzufinden.

Diese Grundbedingungen, die in Wechselwirkung zueinander stehen, markieren einen Kernpunkt professionellen Handelns in Pädagogik und Sozialer Arbeit, nämlich das Verhältnis von Subjekt und Ort. "Es handelt sich einerseits um die Frage, wie Subjektivität als Eigenleistung des Subjekts her- und sichergestellt werden kann, wo sie noch nicht gegeben oder durch die menschlich geschaffenen Umstände selbst verloren ist" (Winkler, 1988, S. 255); andererseits um die Frage, und dies als methodische Priorität, "wie ein Ort beschaffen sein muss, damit ein Subjekt als Subjekt an ihm leben und sich entwickeln kann« (S. 278 f.). 
Dieser Zusammenhang von Subjekt und Ort verweist darauf, dass es eine wichtige professionelle Aufgabe ist, einerseits institutionelle Räume zu schaffen, die den Prinzipien der Prävention, der Integration und der Partizipation verpflichtet sind (BMSFJ 1990, S. 85 ff.); andererseits Räume, in denen die individuellen Verfasstheiten der Individuen berücksichtigt werden, sei es, dass sie als Behinderungen, als entwicklungsbedingte Blockaden, als sozio-kulturelle Verschiedenheiten oder Hochbegabungen in Erscheinung treten. Zum Ansatzpunkt von Bildung, Erziehung und Betreuung werden somit die sozialen, affektiven und kognitiven Potentiale der Individuen, die Wahrnehmung ihrer Differenz vor der Maßgabe ihrer prinzipiellen Gleichheit (Winkler, 2002, S. 66). Dies verweist auch darauf, dass es Subjektivität nur in Kontexten gegenseitiger Anerkennung geben kann (Katzenbach, 2004a).

Triangulierung und Virtualisierung in institutionellen und intersubjektiven Kontexten

Die Topoi von Subjekt und Ort sollen noch etwas weiter vertieft werden. Ich beginne mit dem Ort. In Sozialer Arbeit und Pädagogik wird der Ort unter anderem durch die der fachlichen Arbeit mit den Adressaten vorgelagerten sozialen, kulturellen, finanziellen, gesetzlichen und konzeptionellen Bedingungen, die nur begrenzt durch die Fachkräfte beeinflusst werden können, bestimmt (Krebs u. Müller, 1998). Diese Bedingungen sind Aspekte des Rahmens der konkreten Situationen mit den Adressaten. Sie repräsentieren das, was Winkler (2002) den "dritten Faktor« im Verhältnis zwischen den Fachkräften und den Adressaten nennt. Auf einer Ebene institutioneller Triangulierung bezeichnet dieser Faktor "das strukturelle Dritte“ (Tietel, 2005), auf das sich die beteiligten Akteure mehr oder weniger bejahend, ablehnend oder in kritischer Haltung beziehen. Das strukturelle Dritte der Institutionen, ihre Aufgaben, Regeln und Werte, das auch als Repräsentant der geltenden sozio-kulturellen Ordnung gelten kann, hat eine wichtige Funktion, wenn es auf die Bewältigung der institutionellen Aufgaben ankommt. Es gibt den professionellen Beziehungen einerseits eine spezifische Form und Ausrichtung, muss andererseits aber auch immer wieder erst in der Arbeit mit den 
Adressaten durch die Professionellen zur Geltung gebracht werden. Das strukturelle Dritte wird dadurch auch modifiziert. Diese Form der Triade zwischen institutioneller Aufgabe, Fachkraft und Nutzer in einer kritisch-konstruktiven Weise auszubalancieren, erfordert eine triadische Kompetenz in institutionellen Verhältnissen. Hierbei geht es darum, den Interaktionsraum zwischen den Akteuren der professionellen Situation relativ offen zu halten, so dass einerseits auf der Ebene der Institution und ihrer Regeln sowie andererseits auf der Ebene des interpersonalen Geschehens Bewegungsfreiheit möglich wird. Damit ist die Freiheit zum problemadäquaten Handeln in kritischer Auseinandersetzung mit der Institution und ihren Nutzern gemeint (Tietel, 2005, S. $31 \mathrm{ff}$.).

Das strukturelle Dritte ist der äußere Bezugspunkt professioneller Arbeit, der den Fachkräften Orientierungen bietet, um die Konflikte mit ihren Zielgruppen bewältigen zu können. Die Fachkräfte repräsentieren diese institutionellen Bezugspunkte, sinnvollerweise aber in kritischer Distanz (Körner, 2004, S. 136 ff.). Diese Form einer institutionellen Reflexivität geht noch mit einer anderen Ebene einher, die als nach innen gerichtete, exzentrische Perspektivität auf das interund intrapersonelle Geschehen gekennzeichnet werden kann. Damit sind die Beziehungen interagierender Personen gemeint, die mehr oder weniger trianguliert sein können. Diese Form der Triangulierung repräsentiert einerseits eine internalisierte Struktur im Binnenraum der Personen als auch andererseits einen Modus der Interaktion zwischen den an der professionellen Situation beteiligten Personen. Hier geht es wesentlich um das Erkennen und Auflösen der intra- und intersubjektiven Verstrickungen der Subjekte (Tietel, 2005, S. 31). Gemeinsamer Nenner dieser Bemühungen ist, die Erzeugung und methodische Sicherung eines Spannungsverhältnisses, das heißt eines mzeitweiligen Schwebezustandes zwischen realer und imaginierter Interaktion im pädagogischen Bezug, um darin dem Klienten andere, bessere Handlungsoptionen zu eröffnen " (Körner u. Müller, 2004, S. 133). In diesem Schwebezustand wird es möglich, soziale, institutionelle und individuelle Interaktionsmuster bzw. Bedeutungszuweisungen infrage zu stellen. Die Professionellen erfahren so, wie ihre Klienten oder Schüler ihre Welt deuten, welche Vorannahmen sie dafür einsetzen, welche Gefühle und Konflikte für sie bedeutsam sind, 
ohne dass deswegen die soziale und institutionelle Realität missachtet wird.

Körner und Müller (2004) bezeichnen diese Vorgänge auch als "Virtualisierung", die ermöglichen soll, dass die an der professionellen Interaktion Beteiligten erkennen können, inwieweit die vorgefundene Realität inszeniert und mit welchen Bedeutungen sie aufgeladen wird. Diese Konstruktionen sind Anlass für Verständigungsprozesse zwischen den Adressaten und den Professionellen, da unweigerlich unterschiedliche Interpretationen dieser Situationen aufeinandertreffen und zu vielfältigen Konflikten führen. Die Professionellen als Träger der institutionellen Aufträge und in ihren Funktionen beispielsweise als Lehrer oder als Erziehungsberater instrumentalisieren sich zwecks Erfüllung ihrer beruflichen Aufgaben. Sie stellen in ihrer Person Wissen, sozio-kulturelle und persönliche Lebensentwürfe zur Aneignung zur Verfügung, die Auseinandersetzungen in der unmittelbaren Erziehungs-, Beratungs- und Unterrichtssituation auslösen und die letztlich Konflikte über die gegenwärtigen gesellschaftlichen Umstände darstellen. Diese Konflikte, und dies zeigt die heutige Bildungs- und Erziehungslandschaft sehr deutlich, können nur bedingt durch Macht entschieden werden (Winkler, 2002, S. 65). Es ist daher plausibel, dass die Bildungs-, Erziehungs- und Betreuungsrealität durch vielfältige Anerkennungskämpfe gekennzeichnet ist, die auch auf die normative Grundlage professionellen Handelns verweisen.

Reziproke Anerkennung und die Dreiheit von Bildung, Erziehung und Betreuung

Borst (2004) rückt die Konfliktfähigkeit von Individuen in den Mittelpunkt von Anerkennungskämpfen, denen sie einen Bildungssinn zuweist. In ihrem Verständnis bezieht sich Bildung auf zweierlei: Erstens ist sie eine unhintergehbare Voraussetzung für eine andere Wahrnehmung gesellschaftlicher Sinnkonstituierungen und Deutungsmuster und beinhaltet eine Praxis der Kritik (S. 259), und zweitens ist Bildung nie nur auf die Überlieferung und Aneignung kultureller Sachinhalte reduzierbar, hat nie nur einen psychologisch auf das Individuum bezogenen Sinn, sondern zielt immer auch auf die 
Entwicklung und Veränderung der Menschen und ihrer Identitätsbildung in sozialen Kontexten (S. 263). Dieser Bildungsbegriff bezieht sich auf Erfahrungen bzw. akkumulierte Erfahrungen und ihrer reflexiven Bearbeitung, in der sich die beteiligten Personen selbst verändernd bedenken (Hörster, 2002b, S. 45). Dies ist allerdings nicht nur ein kognitiver Prozess, denn Reflexion und Kooperation in sozialen Zusammenhängen lassen sich nicht trennen von Emotionen und moralischen Gefühlen wie Schuld, Scham, Wut und Kränkung (Göppel, 2003), die besonders im Fall misslingender Anerkennung Triebfedern intersubjektiver Anerkennungskämpfe sind (Honneth, 1990, S. 1052 ff.). Für Anerkennung ist daher charakteristisch, dass sich das Subjekt - und hier liegt eine Koinzidenz zu Bildungsprozessen vor-selbst fremd wird. Damit einher geht eine Erfahrung des Verlusts persönlicher Sicherheiten. Das Wiedererkennen des Eigenen im Fremden ist nur ein Teil von Anerkennung, der andere Teil bezieht sich darauf, dass Anerkennung ihr humanitäres Potential und ihren Bildungssinn nur dort entfalten kann, wo für die Individuen Unbekanntes mit in den Anerkennungsprozess eingeht. Das Risiko des Verlustes persönlicher Sicherheit können Individuen aber nur eingehen, wenn sie wissen, dass ihre elementaren physiologischen und psychologischen Bedürfnisse befriedigt sind und daraus keine existentielle Bedrohungen erwachsen (Winkler, 2006, S. 22; Borst, 2004, S. 264).

Anerkennung ist durch ein labiles Gleichgewicht gekennzeichnet. Wer Anerkennung sucht, ist seinerseits auch immer damit konfrontiert, Anerkennung gewähren zu müssen, um die eigene Anerkennung sicherzustellen. Wechselseitige Anerkennungsprozesse verweisen somit auf eine Austarierung gelingender und misslingender Anerkennung, was sich auch in einem Zusammen- bzw. Auseinanderlaufen von Selbstbehauptung und Abhängigkeit ausdrückt. In diesem Zusammenspiel findet Subjektwerdung statt, jedoch nicht losgelöst von gesellschaftlichen und institutionellen Kontexten und Anpassungszwängen. $\mathrm{Da}$ es in diesem Beitrag nicht um Aspekte der Anerkennung und Anpassung des Subjekts im Allgemeinen geht, möchte ich Prozesse reziproker Anerkennung in institutionellen Kontexten am Beispiel der Schule, hier der Grundschule, und Aspekten der Lehrerinnen-Schüler-Beziehung erläutern. 
Vor dem Hintergrund einer Diskussion über Anerkennung ist die Grundschule deswegen interessant, weil sie eine grundlegende Rolle in der Bildung und Erziehung von Kindern spielt. Zudem ist sie die einzige Schulform in Deutschland, die alle Kinder besuchen und in der sich der gemeinsame Unterricht der Kinder eines Wohngebietes einschließlich der Auffalligen und Behinderten am meisten durchgesetzt hat. Prengel (2005) analysiert das Generationenverhältnis und extrahiert aus diesem drei bestimmende Anerkennungsformen: Egalität, Heterogenität (Differenz) und Hierarchie (S. 18 ff.). Sie transponiert diese Analyse in den institutionellen Kontext der Grundschule und legt für die einzelnen Anerkennungsformen Folgendes dar: Grundlage eines gelingenden und konstruktiven Unterrichts ist erstens die "egalitäre Anerkennung", die auf einer symmetrischen Beziehung zwischen den Kindern und ihren Lehrerinnen fußt. Dies gilt für zwei Aspekte: a) Alle Beteiligten spiegeln sich als Menschen in ihren Bedürfnissen nach Autonomie, Bindung und Fürsorge (Dörr, 2006, S. 63 ff.); und b) nehmen die Kinder ihr gleiches Recht auf Bildung in Anspruch, das Schule bzw. die Lehrerinnen gewährleisten müssen. Zweitens geht es um die differenzierende Anerkennung, das heißt, die Kinder und Lehrerinnen müssen sich als Personen erst kennenlernen, sie sind sich unbekannt, und dazu gehört auch die Sozialisation in die Schülerrolle bzw. die Anpassung der Lehrerrolle an die jeweilige Schülergruppe. Schulspezifisch kommen noch die unterschiedlichen Sachinteressen, die Lerngeschwindigkeiten und das Lernvermögen der Kinder in Auseinandersetzung mit dem Lehrplan zum Tragen. Anzustreben wären in diesem Kontext zielgleiche Bildungsprozesse, wobei jedoch klar sein muss, dass manche Kinder bestimmte Kompetenzstufen nie erreichen werden. Egalität, Differenzierung und Heterogenität können jedoch im schulischen Kontext nicht darüber hinwegtäuschen, dass Schule im gesellschaftlichen Auftrag Kinder auch miteinander vergleichen muss. Drittens kommt daher die Hierarchie ins Spiel, die verschiedenes beinhaltet: Es geht um Bewertung und damit auch um Selektion, es geht auf der professionellen Beziehungsebene aber auch um Respekt und Fürsorge. Gerade letztgenannte Aspekte sind für Bildungs- und Lernprozesse sehr bedeutsam. Konstruktives Lernen gelingt besser, wenn sich die Schüler von einer Person sicher gehalten fühlen, der sie einerseits mehr Wissen und Erfahrung zubilligen, die 
sie andererseits aber auch in ihrer relativen Unwissenheit respektiert, ohne sie zu beschämen (Prengel, 2005; Krebs, 2002).

Für das Bildungs-, Erziehungs- und Betreuungswesen und damit auch für das Schulsystem ist es eine Frage der Zukunftsfähigkeit (Bauer in diesem Band), wie diese Anerkennungsformen gewichtet und in institutionelle Strukturen umgesetzt werden. Das heißt, die Funktion der Schule ist keineswegs gefährdet, wenn egalitäre Formen der Anerkennung und die Anerkennung von Heterogenität vor der Anerkennung der Hierarchie Priorität genießen (Borst, 2003; Trescher, 1991).

Im Zwölften Kinder- und Jugendbericht (BMFSJ, 2005) werden solche Überlegungen aufgegriffen und ein Bildungs- und Erziehungswesen gefordert, dass der Gleichheit und Differenz der Individuen auch auf institutioneller Ebene Ausdruck verleiht (S. 40 f.). Diesem Bericht wird ein erweitertes Bildungsverständnis zugrunde gelegt, "demzufolge aus biographischer Sicht von Kindern alle Lernund Bildungsprozesse ins Blickfeld zu rücken sind, ungeachtet dessen, ob sie als Ergebnis einschlägiger Bildungsinstanzen mit Angebotscharakter oder als Elemente offizieller Lehrpläne des Bildungssystems vorgesehen waren" (S. 26). In diesem Bericht wird dieser Lern- und Bildungsbegriff in der "Trias von Bildung, Erziehung und Betreuung" zusammengefasst, wobei die Verfasser Bildung als eine Art Oberbegriff sehen, in den Erziehung und Betreuung eingelagert sind (S. 26; Datler, 1995, S. 239 ff.). Bildung wird in diesem Bericht nicht allein auf den Erwerb von kognitiven und instrumentellen Kompetenzen reduziert, wobei diesen großes Gewicht beigemessen wird, sondern zielt wesentlich darauf ab, die Subjekte zu befähigen, "sich Zumutungen und Ansprüchen der Gesellschaft, die der individuellen Entfaltung entgegenstehen, zu widersetzen«(S. 31; Klafki, 1996).

\section{Psychoanalytisch-pädagogische Reflexion}

Psychoanalytisch-pädagogisches Handeln und seine Reflexion basiert auf einem breit gefassten integrativen Bildungs- und Entwicklungsverständnis (Trescher, 1988, 1991; Eggert-Schmid Noerr, 2006; Deppe, 2002). Dieses richtet sein Augenmerk nicht nur auf die Heranwachsenden und Erwachsenen, die von sozialer Marginalisie- 
rung, Behinderungen und anderen individuellen Auffälligkeiten betroffen sind, sondern sieht die Notwendigkeit der Herstellung und Unterstützung von Bildsamkeit als eine allgemeine Aufgabe an: Diese impliziert die Förderung instrumenteller und kultureller Kompetenzen, zielt aber gleichermaßen auf soziale und personale Kompetenzen und damit auf intersubjektiv-kommunikative, ästhetisch-expressive, auf Körperlichkeit und die emotionale Innenwelt bezogene Fähigkeiten (Winkler, 2006, S. 32; BMFSJ, 2005, S. 31 ff.).

Innerhalb dieses großen Spektrums richtet psychoanalytisch-pädagogische Reflexion ihr Augenmerk auf besondere Fragen. Der Blick auf das eigene Leben und die Brüche in der Welt- und Existenzerfahrung stehen dabei im Vordergrund: "Mit anderen Worten: In bisher im Dunklen gebliebene soziale Zusammenhänge soll Licht gebracht werden, und Licht gilt traditionell als Metaphorik von Wahrheit und Einsicht" (Dörr, 2006, S. 68). Dieses Sehen ist an die Erfahrungen von intersubjektiver Anerkennung gebunden, das heißt Erfahrungen mit Anderen, die die Vorstrukturen des eigenen expliziten und impliziten Verständnisses sozialer Situationen und persönlicher Fragen infrage stellen. Das psychoanalytische Verstehen von Interaktionsprozessen knüpft an diese paradoxe intersubjektive Grunderfahrung an und forscht nach der individuell-besonderen Bedeutung von Erleben, Verhalten und Denken (Trescher, 1993, S. $169 \mathrm{ff}$.).

Das Spannungsverhältnis zwischen bildender Hilfe zur Lebensbewältigung und Aufarbeitung lebensgeschichtlicher Konfliktlinien ist somit Angelpunkt des professionellen Selbstverständnisses psychoanalytisch-pädagogischer Reflexion. Dieses berührt die Selbstauffassungen der beteiligten Personen einschließlich ihrer belastenden Konflikte und den damit einhergehenden unbewussten Bedeutungen (Gerspach, 2002, S. 147 ff.). Damit diese Konflikte und die sich daraus ergebenden Verstrickungen, die im Übrigen in allen zwischenmenschlichen Begegnungen auftreten, professionell bearbeitet werden können, bedürfen Fachkräfte spezifisch psychoanalytischpädagogischen Wissens und entsprechender Kompetenzen (Müller, Krebs, Finger-Trescher, 2002).

Ein Baustein dieses Konzeptes ist der psychoanalytische Topos, dass das Sich-einbeziehen-Lassen in die professionellen Interaktionen Ausgangspunkt und Weg der Konfliktbewältigung darstellt, der 
"methodisch für den Erkenntnisgewinn unerlässlich ist" (Trescher, 1993, S. 168). Die Erkenntnis und das Verstehen wird durch die schrittweise Triangulierung der professionellen Interaktionen gesichert und basiert auf der Virtualisierung festgefahrener Konfliktmodi, die unter anderem auf unerkannten Übertragungen und Gegenübertragungen beruhen.

Unter Übertragung wird ein ungelöster, innerer lebensgeschichtlicher Konflikt verstanden, der sich innerhalb der professionellen Beziehung in einen intersubjektiven Konflikt verwandelt. Die andere Person, hier der Professionelle, wird sozusagen als Stellvertreter früherer Beziehungspartner gesehen. Die Szenen können sich so aufgliedern, dass entweder Erfahrungen mit einem Anderen, beispielsweise Vater oder Mutter, übertragen werden, oder Aspekte und Erlebnisweisen, die mehr die eigene Person, die eigenen Selbsterfahrungen betreffen. In der Regel sind beide Anteile gleichzeitig virulent, jedoch unterschiedlich gewichtet (Altmeyer u. Thomä, 2006; Herold u. Weiß, 2002). "Diese unbewussten Übertragungsreaktionen äußern sich in konkreten, wenn auch verschlüsselten Interaktionsformen und Beziehungsangeboten " (Finger-Trescher, 2001, S. 1457), denen unabgegoltene sexuelle und aggressive Regungen, Gefühle und Affekte wie beispielsweise Trauer, Schuld, Scham und Kränkungen zugrunde liegen. Diese Angebote, Wünsche und Bedürfnisse signalisieren dem professionell Beteiligten, wie der Übertragende die Beziehung zu ihm gestalten möchte, und es werden entsprechende Gegenübertragungsreaktionen ausgelöst. Diese können auch als "Übertragungsidentifizierungen" bezeichnet werden. Sie entstehen in dynamischen Prozessen zwischen zwei oder mehreren Personen, die sich im Kontext pädagogischer und sozialer Felder - aus dem Zusammenspiel von Übertragung und Gegenübertragung - zu "belastungstypischen Konflikten und Szenen" (Trescher, 1993) verdichten. Hier kommt dann eine spezifische psychoanalytische Wahrnehmungshaltung zum Tragen, die allerdings angesichts gelingendem professionellen Handeln in den Hintergrund tritt. In einer Pendelbewegung zwischen Letztgenanntem und den irritierenden, belastenden und sich wiederholenden Konflikten werden durch die Analyse der Gegenübertragungsreaktionen und über Probeidentifizierungen durch Hypothesenbildungen die Grenzen des Verstehens schrittweise ausgedehnt (S. 193). 
Diesem Unterfangen stellen sich aber in der Regel erhebliche emotionale und kognitive Widerstände entgegen. Um damit umgehen zu können, schlägt Körner (1996) vor, die professionellen Beziehungen in den Feldern der Pädagogik und Sozialen Arbeit als eine Art Probebühne anzusehen. Wirksame Veränderungen im Hinblick auf interpersonelle Verstrickungen und Entwicklungsblockaden beruhen weniger auf lebensgeschichtlichen Rekonstruktionen, sondern darauf, dass die professionell Beteiligten den Adressaten ihr eigenes Verständnis der gemeinsamen Situationen anbieten. Dies muss sich innerhalb eines Dialoges zwischen Personen ereignen, die füreinander bedeutungsvoll geworden sind. Das heißt, aus einem Geschehen, das vielleicht zu Beginn einer professionellen Beziehung noch als AktionReaktion von Einzelpersonen gesehen werden kann, muss etwas reziprok Gemeinsames entstanden sein. Die Einigung auf dieses Gemeinsame, das in Teilen auch differente Interpretationen einschließt, ist deswegen wirksam, weil innere Konflikte durch Empathie und Identifizierung erfahrbar und reflexiv nachvollziehbar werden. Sie können in der Einigung - auf der Basis eines dialogischen Verhältnisses - integriert werden. Allerdings hängt diese modifizierende Integration einerseits von der Intensität der mit diesen Konflikten verbundenen Ängste der Adressaten ab und basiert andererseits auf der Fähigkeit der Professionellen, genügend unbefangen mit diesen Konfliktanteilen umgehen zu können (Trescher, 1993, S. 173 ff.; Krebs, 2006; Katzenbach, 2004b).

An dieser Stelle kommt aber noch ein weiteres kritisches Korrektiv zur Wirkung. Die Professionellen müssen unbedingt darauf achten, dass sie weder ihren eigenen Übertragungsbereitschaften noch denen ihrer Gegenüber erliegen, obwohl diese im professionellen Prozess immer auch ein Stück weit ausagiert werden. Trescher (1993) formuliert diesen Sachverhalt wie folgt:

"Sich verwenden lassen, den anderen nicht verwenden. Dies impliziert zuallererst den Verzicht auf die Befriedigung eigener Wünsche durch die Klienten, aber auch die Verweigerung der Befriedigung bestimmter Wünsche der Klienten" (S. 182).

Der wechselseitigen Manipulation wäre andernfalls Tür und Tor geöffnet. Das heißt, diese intersubjektiv-kommunikative Konzeption 
der professionellen Beziehungen basiert auf einem spezifischen Begriff reflexiver Abstinenz. Dieser schließt aktives Handeln und auf die Adressaten zugehendes Verhalten nicht aus. Abstinenz ist vielmehr als prinzipielle Haltung so zu verstehen, dass Reflexion und Metakommunikation immer wieder neu erarbeitet werden müssen. Aus egozentrischen, durch unbewältigte Konflikte eingeengten Beziehungsformen (Gerspach, 2006, S. $12 \mathrm{ff}$.) können so triangulierte Beziehungsformen entstehen, in denen alle am professionellen Geschehen Beteiligten, ihre Rollen, ihre Beziehungen, ihre Lebenserfahrungen und Wünsche angemessener erfahren und erkennen können. So soll das bisher Unabgegoltene vergangener und gegenwärtiger bewusster, aber auch unbewusster Konflikte mit einer Zukunftsperspektive zum Tragen kommen können.

Diese grundlegende Reziprozität der professionellen Beziehungen setzt nicht die Vorgabe außer Kraft, dass die Fachkräfte aufgrund ihrer Aufgabenprofile für die Bildungs- und Entwicklungsinteressen der Adressaten eintreten. Die damit verbundene Parteilichkeit und Asymmetrie ist aber nur dann Garant für einen Freiheitsspielraum im Hinblick auf selbstbestimmtere und weniger durch Zwänge geprägte Umgangformen im Bildungs-, Sozial- und Erziehungswesen, wenn sie mit dem Konzept einer Grenze der Verfügbarkeit der Subjekte verkoppelt werden. Diese wird im Begriff der intersubjektiven Anerkennung thematisch, der eine normative Grundlage für professionelles Handeln zur Verfügung stellt (Katzenbach, 2004a; Honneth, 1997). Menschen werden nur aus der Perspektive eines zustimmenden Anderen zu handlungsfähigen Subjekten, die sich selbst achten und vertrauen (Honneth, 1990, S. 1048). Bleiben solche zustimmenden Reaktionen dauerhaft aus, werden sie durch soziale Marginalisierung und andere sozialisationsbedingte Nachteile chronisch, dann reißt dies Lücken in die Persönlichkeiten der Betroffenen und unterläuft gelingende Bildungs- und Entwicklungsprozesse. Gleichwohl enthalten diese Missachtungserfahrungen allerdings auch das Potential, dass die betroffenen Subjekte sich gegen die ihnen zugefügten Benachteiligungen zur Wehr setzen. Es wäre dann die Aufgabe von Professionellen, sie hierin zu unterstützen und darauf abgestimmte Bildungs- und Entwicklungsangebote zu machen (Borst, 2003, S. 179 ff.; Dörr, 2006, S. 70 f.). Mit der Einnahme dieses normativen Standpunktes sollen die intersubjektiven Bedingungen 
gesichert werden, die es menschlichen Subjekten erlauben, ihre Integrität zu wahren.

\section{Literatur}

Ahrbeck, B. (2004). Kinder brauchen Erziehung. Die vergessene pädagogische Verantwortung. Stuttgart: Kohlhammer.

Altmeyer, M., Thomä, H. (2006). Einführung: Psychoanalyse und Intersubjektivität. In M. Altmeyer, H. Thomä (Hrsg.), Die vernetzte Seele. Die intersubjektive Wende in der Psychoanalyse (S. 7-31). Stuttgart: Klett-Cotta.

BMFSJ - Bundesministerium für Familie, Senioren, Frauen und Jugend (1990). Achter Jugendbericht. Bundesdrucksache 11/6576. Bonn: Eigenverlag.

BMFSJ - Bundesministerium für Familie, Senioren, Frauen und Jugend (BMSFJ) (2005). 12. Kinder- und Jugendbericht: Bildung, Betreuung und Erziehung vor und neben der Schule. Bundesdrucksache 15/6014. Berlin. Zugriff am 13.8.2007 unter www.bmfsj.de.

Borst, E. (2003). Anerkennung des Anderen und das Problem des Unterschieds. Perspektiven einer kritischen Theorie der Bildung. Baltmannsweiler: Schneider-Verlag.

Borst, E. (2004). "Anerkennung" als konstitutives Merkmal sozialpädagogischer Bildungsvorstellungen. Neue Praxis. Zeitschrift für Sozialarbeit, Sozialpädagogik und Sozialpolitik, 34 (3), 259-270.

Datler, W. (1996). Bilden und Heilen. Auf dem Weg zu einer pädagogischen Theorie psychoanalytischer Praxis. Mainz: Grünewald.

Deppe, H. (2002). Integration und Solidarität. In B. Warzecha (Hrsg.), Zur Relevanz des Dialogs in Erziehungswissenschaft, Behindertenpädagogik, Beratung und Therapie (S. 39-58). Hamburg: LIT-Verlag.

Deutsches PISA-Konsortium (Hrsg.) (2001). PISA 2000. Basiskompetenzen von Schülerinnen und Schülern im internationalen Vergleich. Opladen: Leske + Budrich.

Dörr, M. (2006). Der unterstützende Blick auf das eigene Leben. Zur Paradoxie von Freiheit und Zwang reflexiver Lebensplanung. In V. Fröhlich, R. Göppel (Hrsg.), Bildung als Reflexion über Lebenszeit (S. 62-74). Gießen: Psychosozial-Verlag.

Eggert-Schmid Noerr, A. (2006). Bildung und Soziale Arbeit. In H. Frohnhofen, S. Schweior-Popp (Hrsg.), Bildung. Schriftenreihe der KFH Mainz. Bd. 1 (S. 172-190). St. Ottilien: EOS Verlag.

Finger-Trescher, U. (2001). Psychoanalytische Sozialarbeit. In. H. U. Otto, H. Thiersch (Hrsg.), Handbuch Sozialarbeit/Sozialpädagogik (S. 1454-1461). Neuwied: Luchterhand. 
Gerspach, M. (2002). Der Beitrag der Psychoanalysezum Dialog. In B. Warzecha (Hrsg.), Zur Relevanz des Dialogs in Erziehungswissenschaft, Behindertenpädagogik, Beratung und Therapie (S. 125-168). Hamburg: LIT-Verlag.

Göppel, R. (2003). "Die Kultur der Affekte ist das eigentlich schwerste Bildungsziel" (A. Mitscherlisch) - Möglichkeiten, Probleme und Grenzen einer "Bildung der Gefühle«. In M. Dörr, R. Göppel (Hrsg.), Bildung der Gefühle. Innovation? Illusion? Intrusion? (S. 15-39). Gießen: Psychosozial-Verlag. Helsper, W., Hörster, R., Kade, J. (Hrsg.) (2003). Ungewissheit. Pädagogische Felder im Modernisierungsprozess. Weilerswist: Velbrück-Wissenschaft.

Herold, R., Weiß, H. (2002). Übertragung. In W. Mertens, B. Waldvogel (Hrsg.), Handbuch psychoanalytischer Grundbegriffe (2. Aufl.) (S. 758771). Stuttgart: Kohlhammer.

Honneth, A. (1990). Integrität und Missachtung. Grundmotive einer Moral der Anerkennung. Merkur. Deutsche Zeitschrift für europäisches Denken, 44, (501), 1043-1054.

Honneth, A. (1997). Anerkennung und moralische Verpflichtung. Zeitschrift für philosophische Forschung, 51, (1), 25-41.

Hörster, R. (2002a). Pädagogisches Handeln. In H. H. Krüger, W. Helsper (Hrsg.), Einführung in Grundbegriff und Grundfragen der Erziehungswissenschaft (S. 35-42). Opladen: Leske + Budrich.

Hörster, R. (2002b). Bildung. In H.H. Krüger, W. Helsper (Hrsg.), Einführung in Grundbegriff und Grundfragen der Erziehungswissenschaft (S. 43-52). Opladen: Leske + Budrich.

Katzenbach, D. (2004a). Anerkennung, Missachtung, und geistige Behinderung. Sozialphilosophische Perspektiven aufden so genannten Paradigmenwechsel in der Behindertenpädagogik. In B. Ahrbeck, B. Rauh (Hrsg.), Behinderung zwischen Autonomie und Angewiesensein (S. 127-144). Stuttgart: Kohlhammer.

Katzenbach, D. (2004b). Das Problem des Fremdverstehens. Psychoanalytische Reflexion als Beitrag zur Professionalisierung geistigbehindertenpädagogischen Handelns. In E. Wüllenweber (Hrsg.). SozialeProbleme von Menschen mit geistiger Behinderung. Fremdbestimmung, Benachteiligung, Ausgrenzung und soziale Abwertung (S. 322-334). Stuttgart: Kohlhammer.

Kerstan, Th. (2001). Ein lehrreiches Desaster. Das deutsche Bildungssystem hat versagt: Es ist ungerecht und produziert Mittelmaß - das zeigt die internationale Schulstudie PISA. Die Zeit (50), 45-46.

Klafki, W. (1996). Die Bedeutung der klassischen Bildungstheorien für ein zeitgemäßes Konzept allgemeiner Bildung. In W. Klafki, Neue Studien zur Bildungstheorie und Didaktik. Zeitgemäße Allgemeinbildung und kritischkonstruktive Didaktik (5. Aufl.) (S. 15-42). Weinheim: Beltz.

Klemm, K. (2004). Die Hauptschule bleibt sitzen. Auch die neue PISA-Studie zeigt, dass die Wahl der Schulform von der sozialen Herkunft der Schüler abhängt. Frankfurter Rundschau (287), 7. 
Körner, J. (1996). Zum Verhältnis pädagogischen und therapeutischen Handelns. In A. Combe, W. Helsper (Hrsg.), Pädagogische Professionalität. Untersuchungen zum Typus pädgogischen Handelns (S. 780-809). Frankfurt a. M: Suhrkamp.

Körner, J. (2004). Die trianguläre Situation in der Psychoanalyse und der psychoanalytischen Pädagogik. In R. Hörster, E. W. Küster, St. Wolff (Hrsg.), Orte der Verständigung. Beiträge zum sozialpädagogischen Argumentieren (S. 126-138). Freiburg i. Brsg.: Lambertus.

Körner, J., Müller, B. (2004). Chancen der Virtualisierung - Entwurf einer Typologie psychoanalytisch-pädagogischer Arbeit. In. W. Datler, B. Müller, U. Finger-Trescher (Hrsg.), Themenschwerpunkt: Sie sind wie Novellen zu lesen ... Zur Bedeutung von Falldarstellungen in der Psychoanalytischen Pädagogik. In Jahrbuch für Psychoanalytische Pädagogik (Bd. 14) (S. 132151).Gießen: Psychosozial-Verlag.

Krebs, H. (2002). Emotionales Lernen in der Schule - Aspekte der Professionalisierung von Lehrerinnen und Lehrern. In B. Müller, U. Finger-Trescher, H. Krebs (Hrsg.), Themenschwerpunkt: Professionalisierung in sozialen und professionellen Feldern. Impulse der Psychoanalytischen Pädagogik. Jahrbuch für Psychoanalytische Pädagogik (Bd. 13) (S. 47-69). Gießen: Psychosozial-Verlag.

Krebs, H. (2006). Psychoanalytisch-pädagogische Erziehungsberatung. Zwei Fallbeispiele. In B. Ahrbeck, B. Rauh (Hrsg.), Der Fall des schwierigen Kindes. Therapie, Diagnostik und schulische Förderung verhaltensgestörter Kinder und Jugendlicher (S. 140 -159). Weinheim: Beltz.

Krebs, H., Müller, B. (1998). Der psychoanalytische Begriff des Settings und seine Rahmenbedingungen im Kontext der Jugendhilfe. In B. Müller, U. Finger-Trescher, H. Krebs (Hrsg.). Themenschwerpunkt: Jugendhilfe und Psychoanalytische Pädagogik. Jahrbuch für Psychoanalytische Pädagogik (Bd. 9) (S. 15-40). Gießen: Psychosozial-Verlag.

Müller, B. (1994). Sozialpädagogisches Können : ein Lehrbuch zur multiperspektivischen Fallarbeit (2. Aufl.). Freiburg i. Brsg.: Lambertus.

Müller, B. (2001): Methoden. In H. U. Otto, H. Thiersch (Hrsg.), Handbuch Sozialarbeit/Sozialpädagogik (S. 1194-1204). Neuwied: Luchterhand.

Müller, B., Krebs, H., Finger-Trescher, U. (2002). Professionalisierung in sozialen und pädagogischen Feldern. Impulse der Psychoanalytischen Pädagogik. In B. Müller, U. Finger-Trescher, H. Krebs, (Hrsg.), Themenschwerpunkt: Professionalisierung in sozialen und professionellen Feldern. Impulse der Psychoanalytischen Pädagogik. Jahrbuch für Psychoanalytische Pädagogik (Bd. 13) (S. 9-26). Gießen: Psychosozial-Verlag.

Prengel, A. (2005). Anerkennung von Anfang an - Egalität, Heterogenität und Hierarchie im Anfangsunterricht und darüber hinaus. In U. Geiling, A. Hinz (Hrsg.), Integrationspädagogik im Diskurs. Auf dem Weg zu einer inklusiven Pädagogik (S. 15-34). Bad Heilbrunn: Klinkhardt. 
SGB - Sozialgesetzbuch. Achtes Buch. Kinder- und Jugendhilfe. Berlin. Zugriff am 5.12.2005 unter www.bmfsj.de.

Tietel, E. (2005). Institutionelle Triangulierung aus psychoanalytischer und systemischer Sicht. In Triangel-Institut (Hrsg.), Brücken und Tücken psychoanalytisch-systemischer Beratung (2. Aufl.) (S. 24-44). Berlin: Ulrich Leutner Verlag.

Trescher, H. G. (1988). Erziehungswissenschaft und Psychoanalyse. Neue Praxis, (6), 455-464.

Trescher, H. G. (1991). „Ungleichheit für alle! - Aspekte des Gegenstandes, der Methode und der Lehre psychoanalytischer Heilpädagogik. Zeitschrift für Hochschuldidaktik, 15, (3), 324-346.

Trescher, H. G. (1993). Handlungstheoretische Aspekte der Psychoanalytischen Pädagogik. In M. Muck, H. G. Trescher (Hrsg.), Grundlagen der Psychoanalytischen Pädagogik (S. 167-201). Mainz: Grünewald.

Winkler, M. (1988). Eine Theorie der Sozialpädagogik. Stuttgart: Klett.

Winkler M. (2002). Erziehung. In H. H. Krüger, W. Helsper(Hrsg.), Einführung in Grundbegriff und Grundfragen der Erziehungswissenschaft (S. 53-72). Opladen: Leske + Budrich.

Winkler, M. (2006). Lernen und vergessen - wie die späte Moderne die Bedingungen des Aufwachsens verändert. In A. Eggert-Schmid Noerr, U. Pforr, H.Voß-Davies (Hrsg.), Lernen, Lernstörungen und die pädagogische Beziehung (S. 13-37). Gießen: Psychosozial-Verlag. 